\title{
Hybrid expert system and simulated annealing approach to optimal reactive power planning
}

\author{
W.-S JWo \\ C. - W. Liu \\ C.-C. Liu
}

Y.-T. Hsiao

Indexing terms: Reactive power planning, Expert systems, Simulated annealing, VAR

\begin{abstract}
The reactive power source planning problem has a significant influence on secure and economic operation in electric power systems. To achieve both goals, system maximum security and minimum cost in operation, reactive power planning is posed as a multiobjective optimisation problem in terms of mathematical language. In the paper the authors present an effective algorithm based on hybrid expert system simulated annealing (ESSA) to circumvent the complicated planning problem. A more practical problem formulation with multiobjectives and constraints is presented. Then, ESSA is introduced to search the global optimal solution considering both quality and speed at the same time. Simulation cases are used to evaluate the proposed algorithm.
\end{abstract}

\section{Introduction}

Recently, due to environmental and economical pressures to force system operation closer to the stability boundary, the VAR planning problem has attracted a great deal of attention from both industry and academics. In the past two decades the goal of VAR planning has been focused on providing enough reactive power, by installing VAR sources in the bus, to minimise overall real power loss and maintain voltage profiles within specified levels.

Because of this goal, VAR planning has commonly been formulated as a complicated constrained optimisation problem with partially discrete, partially continuous and nondifferentiable nonlinear objective functions [1-6]. A survey of the literature on the problem reveals that various numerical optimisation techniques have been employed to approach the complicated VAR planning problem. More specifically, Opoku [3] has formulated the problem as a mathematical optimisation problem based on a linearised model (a restructured sparse admittance matrix) to reduce dimensionality and computing time. Lebow [1], Granville [2] and Hong [4]

\section{(IEE, 1995}

Paper 1958C (P9, PII), first received 18th October 1994 and in fina revised form 16th March 1995

Wu-Shun Jwo, Chih-Wen Liu and Chun-Chang Liu are with the Department of Electrical Engineering, National Taiwan University, Taipei, Taiwan, Republic of China

Ying-Tung Hsiao is with the Department of Electrical Engineering, Tamkang University, Taipei, Taiwan, Republic of China

IEE Proc.-Gener. Transm. Distrib., Vol. 142, No. 4, July 1995 have fomulated the problem as a mixed-integer nonlinear programming problem with $0-1$ integer variables representing whether new reactive devices should or should not be installed. In this formulation, however, both the number and value of capacitors were still treated as continuously differentiable. The Genders Benders Decomposition (GBD) technique [7] was then employed to decompose the problem into a continuous subproblem and an integer subproblem. It should be noted that the above-mentioned methods can be classified as a greedy search technique. One main disadvantage of the aforementioned techniques is that they often get stuck at local rather than at global optima. To circumvent this problem, Hsiao et al. [5, 6] applied the simulated annealing (SA) method to optimal VAR source planning in large-scale power systems. SA is a powerful, generalpurpose optimisation technique, which can theoretically converge asymptotically to the global optimum solution with probability l. One main drawback, however, of SA is that it takes much CPU time to find the global optimum.

In this paper, we present a hybrid expert-system/ simulated-annealing method to improve the CPU time of SA while retaining the main characteristics of SA, i.e. the ability to obtain the global optimal solution. A new formulation of the optimal VAR planning as a constrained multiobjective optimisation problem is presented in Section 2. Then a fuzzy satisfying method is described for solving general multiobjective optimisation problems in Section 3. A detailed hybrid expert-system/simulatedannealing algorithm is described in Section 4. In Section 5 the proposed algorithm is implemented in a software package and tested on an IEEE 30-bus system, and promising results are obtained. Also, the performances of various membership functions of each objective are compared in this Section. Finally, a summary conclusion is given in Section 6.

\section{Problem formulation}

In this Section a new formulation of the optimal VAR planning problem, formulated as a constrained, nondifferentiable, multiobjective optimisation problem, is presented.

\subsection{Objective functions}

There are two objective functions to be considered in this problem. The first function, expressed in eqn. 1, represents the cost of VAR source placements plus the total cost of energy loss. The second function, expressed in 
eqn. 2 , is the measure of voltage deviation in the system:

$$
\begin{aligned}
& f_{1}(x)=\sum_{i=1}^{m}\left[C_{i}(x)+k_{e} D_{i} P_{\text {Loss }, i}\right] \\
& f_{2}(x)=\max _{k} \frac{\left|V_{k}-V_{k r}\right|}{V_{k r}}
\end{aligned}
$$

where

$x=$ expansion variable vector of the new VAR sources

$k_{e}=$ energy cost per unit $(\$ / \mathrm{kWh})$

$D=$ duration of load level $i$

$P_{\text {loss }}=$ real power loss of load level $i$ in the system

$C_{i}=$ total purchase cost of load level $i$ of new installment VAR sources, and

$C_{i}=\sum_{k \in \Omega}\left(d_{k}^{i}+s_{c k}^{i} q_{c k}^{i}+s_{r k}^{i} q_{r k}^{i}\right) r_{k}^{i} \quad(i=1,2, \ldots, m)$

$m=$ total number of buses in the system

$\Omega=$ a set of all candidate buses to install new VAR sources

$d_{k}^{i}=$ installment cost at bus $k$

$s_{c k}^{i}=$ unit costs of capacitor

$s_{r k}^{i}=$ unit costs of reactor

$q_{c k}^{i}=$ added capacitive compensation of load level $i$ at bus $k$

$q_{r k}^{i}=$ added inductive compensation of load level $i$ at bus $k$

0 don't placement reactive power source at

$r_{k}^{i}=$ bus $k$ 1 placement reactive power source at bus $k$

$v_{k}=$ voltage magnitude at bus $k$

$v_{k r}=$ ideal voltage magnitude at bus $k$

\subsection{Load and operational constraints}

The load constraints described by a set of power flow equations results from the conservation of real and reactive power. In vector form, the overall power flow equations is expressed as

$$
L(x)=0
$$

The following operational constraints are considered:

(i) line flow limits

(ii) voltage magnitudes and phase angle difference limits

(iii) transformer tap charging limits

(iv) real and reactive power generations limits, and

(v) reactive power compensations limits.

These constraints are expressed in the following vector form:

$$
\boldsymbol{G}(x) \leqslant 0
$$

\subsection{Overal problem}

In summary, the multiobjective VAR planning problem is formulated as the following form:

$$
\operatorname{Min} f_{1}(x)
$$

$\operatorname{Min} f_{2}(x)$

subject to

$$
\begin{aligned}
& L(x)=0 \\
& G(x) \leqslant 0
\end{aligned}
$$

\section{Fuzzy satisficing method for multiobjective
optimisation problem}

In this Section we begin by introducing a fuzzy satisficing method [9] for solving a general multiobjective optim- isation problem, which will be used in the development of the presented ESSA algorithm. Basically, the ideal of the fuzzy satisficing method is that the complicated multiobjective optimisation problem is transformed to a single minimax optimisation problem by virtue of assigning each objective function with a fuzzy goal by the decision maker (DM). The fuzzy goals are quantified by selected membership functions and reference membership values.

In what follows, the fuzzy satisficing method is described in more detail. Consider a general multiobjective optimisation problem:

$$
\begin{gathered}
\min f_{1}(\boldsymbol{x}) \\
\min f_{2}(\boldsymbol{x}) \\
\vdots \\
\min f_{k}(\boldsymbol{x})
\end{gathered}
$$

subject to

$$
g_{m}(x) \leqslant 0 ; m=1,2, \ldots, n \quad x \in S
$$

where $x$ is a vector of decision variables, $f_{x}(x)$ is the $k$ th distinct objective function of the decision vector $x, g_{m}(x)$ is the $m$ th inequality constraint, and $S$ is the feasible solution set.

Assume that the DM has imprecise or fuzzy goals for each objective function in the multiobjective optimisation problem. We first decide a membership function $u\left(f_{i}(\boldsymbol{x})\right)$ for each of the objective functions $f_{\mathrm{i}}(\boldsymbol{x}), i=1,2, \ldots, k$. Here it is assumed that $u\left(f_{i}(x)\right)$ is a strictly monotone decreasing and continuous function with respect to $f_{i}(\boldsymbol{x})$ and $u\left(f_{i}(x)\right)=0$ or $\rightarrow 0$ if $f_{i}(x) \geqslant f_{i}^{0}$ and $u\left(f_{i}(x)\right)=1$ or $\rightarrow 1$ if $f_{i}(x) \leqslant f_{i}^{1}$, where $f_{i}^{\circ}$ is an unacceptable level for $f_{i}(x)$ and $f_{i}^{i}$ is a totally desirable level. Namely, $u\left(f_{i}(x)\right)$ is defined [9] by

$$
u\left(f_{i}(x)\right)= \begin{cases}1 \text { or } \rightarrow 1, & \text { if } f_{i}^{1}>f_{i}(x) \\ d_{i}\left(f_{i}(x)\right), & \text { if } f_{i}^{1} \leqslant f_{i}(x) \leqslant f_{i}^{0} \\ 0 \text { or } \rightarrow 0, & \text { if } f_{i}(x)>f_{i}^{0}\end{cases}
$$

where $d_{i}\left(f_{i}(x)\right)$ is a strictly monotone decreasing and continuous function with respect to $f_{i}(\boldsymbol{x})$. For example, the linear, exponential and piecewise-linear membership functions are shown in Figs. $1 a, b$ and $c$, respectively.

After determining the membership function for each of the objective functions, the DM is asked to specify his reference levels of achievement of the membership functions, called reference membership values, $i=1, \ldots, k$. Hence the problem of eqn. 6 is transformed into eqn. 8 , a fuzzy multiobjective decision problem or a single minimax optimisation problem.

$$
\operatorname{Min}_{x \in S} \operatorname{Max}\left(\bar{u}_{f_{i}}-u\left(f_{i}(x)\right)\right)
$$

or, equivalently,

$$
\operatorname{Min}_{v, x \in S}
$$$$
v, x \in S
$$

where

$$
v=\operatorname{Max}_{1 \leqslant i \leqslant k}\left(\bar{u}_{f_{i}}-u\left(f_{i}(x)\right)\right)
$$

To sum up, the algorithm of the fuzzy satisficing method can be described as below:

Step $I$ : (individual unacceptable and desirable level): Decide the individual unacceptable level $f_{i}^{0}$ and desirable level $f_{i}^{1}$ of each objective function $f_{i}(\boldsymbol{x})$.

Step 2: (membership functions): Use a suitable membership function $u\left(f_{i}(x)\right)$ for each of the objective functions.

IEE Proc.-Gener. Transm. Distrib., Vol. 142, No. 4, July 1995 
Step 3: (reference membership values): Set the reference membership values $\bar{u}_{f_{i}}, i=1, \ldots, k$.

Step 4: Solve the single minimax optimisation problem, eqns. 8 or 9 .
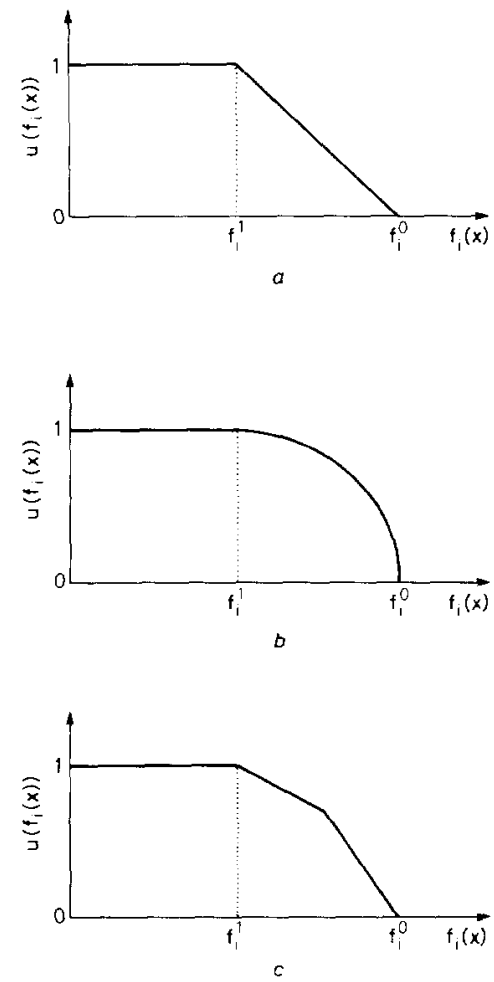

Fig. 1 Three types of membership function

a Linear

$b$ Exponential

c Piecewise-linea

\section{Hybrid expert-system/simulated-annealing algorithm}

The two-staged optimisation method, ESSA, shown in Fig. 2, containing an expert system and a simulated annealing technique, is presented for solving multiobjective VAR planning, eqn. 5. In the first stage of

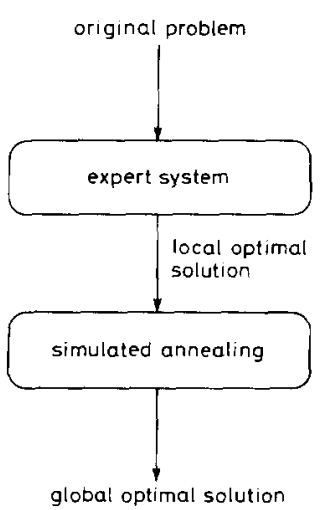

Fig. 2 Basic configuration of ESSA algorithm

IEE Proc.-Gener. Transm. Distrib., Vol. 142, No. 4, July 199
ESSA, we use an expert system consisting of several heuristic rules to find a local optimal solution, which will be employed as an initial starting point of the second stage. Then we apply a simulated annealing technique to transformed single minimax optimisation from an original multiobjective optimisation problem based on the fuzzy satisficing method.

\subsection{First stage: expert system}

In the first stage, the expert system consists of a heuristic rule base. A nonlinear objective function is used as the optimisation goal and a power loss sensitivity index (SI) vector is defined as the guide process of solving the optimal solution.

The heuristic algorithm can be described as below:

Step I: To run load flow program. This obtains voltage magnitudes and phase angles of all buses in the power system.

Step 2: Calculate power loss sensitivity index $S I$ [8]. This quantifies information on the extent of impact on placing an incremental reactive power $\Delta Q_{i}$ at bus $i$ on power loss reduction.

$$
S I=\left[\frac{\partial P_{L}}{\partial Q_{1}}, \frac{\partial P_{L}}{\partial Q_{2}}, \ldots, \frac{\partial P_{L}}{\partial Q_{i}}, \ldots, \frac{\partial P_{L}}{\partial Q_{n}}\right]
$$

where $\left(\partial P_{L} / \partial Q_{i}\right)$ is the power loss sensitivity index of bus $i, P_{L}$ is the total power loss in the system, and $Q_{i}$ is the add reactive power source at bus $i\left(Q_{i}\right.$ is positive for the capacitor).

Step 3: To create a list, a set of all candidate buses to install new VAR sources is called 'CANDIDATE' according to heuristic rule 1 (HR1). HR 1 is used to select all candidate buses, then put them into CANDIDATE

Step 4: If CANDIDATE is empty, then run the terminating rule.

Step 5: Select the $i$ th bus on CANDIDATE according to heuristic rule set 2 (HR2). HR2 is used to select a bus to be placed in an unit reactive power source, or the bus is eliminated from CANDIDATE.

Step 6: If CANDIDATE is empty, then run the terminating rule. 5

Step 7: If CANDIDATE is not empty, then go to step

In the above algorithm, HR1, HR2 and the terminating rule are explained in the Appendix.

\subsection{Second stage: simulated annealing}

The second stage uses a simulated annealing technique for the optimal reactive power planning problem. Simulated annealing is a powerful general-purpose technique for solving combinatorial optimisation problems. This technique is an approach that attempts to avoid entrapment in poor local optima by allowing an occasional uphill move. This is done under the influence of a random number generator and a control parameter called the temperature. It has been shown that this technique converges asymptotically to the global optimal solution with probability ' 1 ' $[10,11]$.

The algorithm of SA at each 'temperature' $T$ is briefly described as below (for more details see [6]) :

repeat \{
1. perturb
2. evaluate cost
3. accept/update
until stop criterion $=$ true 
Step 1: Perturb the current system configuration (S) to a new configuration ( $\mathrm{S}^{\prime}$ ).

Step 2: Evaluate the change of the cost function $\Delta c=\cos t\left(\mathbf{S}^{\prime}\right)-\operatorname{cost}(\mathbf{S})$.

Step 3: Accept/update: If the move decreases the value of the cost function, i.e. $\Delta c<0$, the move is accepted and the new configuration is refined. On the other hand (when $\Delta c>0$ ), if $\exp (\Delta c / T)>a$ random number $r \in[0$, 1), the uphill move is accepted; otherwise the move is used for the next step. Physically, this means that the system will accept uphill moves with reasonable probability as long as these moves do not increase the cost by more than $T$. It is due to the probabilistic selection rule that the process can always get out of a local optimum and proceed to the desired global optimum.

The simulated annealing technique and $\varepsilon$-constraint method have been applied to the VAR planning problem with the following features [6]

(i) It allows a more realistic problem formulation.

(ii) It has the ability to handle the multiobjective problem.

(iii) It allows a tradeoff choice made by the planners.

However, its requirement for computation time grows rapidly by system size. The computation time of simulated annealing becomes prohibitive for a large-scale power system. Also, the tradeoff tolerance of the $\varepsilon$ constraint method plays a critical role in their solution algorithms. Selecting a proper tradeoff tolerance is quite dependent on the good judgments and experiences of a planner. To overcome these problems, we present in the paper a hybrid expert system (instead of the $\varepsilon$-constraint method) and the simulated annealing algorithm to reduce the run time of the process and incoroporate experiences of experts into the solution algorithm.

\section{Numerical results}

In this Section an IEEE 30-bus system was tested. The system was modified as: generator Q-limit $(\sim 0 \sim$ 30 Mvar), the tap operational limit $(\sim 0.95 \sim 1.05 \%)$ and reactive power loads are twice the original value.

In the IEEE 30-bus system, the membership functions of the cost function $f_{1}$ and the voltage deviation function $f_{2}$ are linear, as shown in Fig. $1 a$, and set the reference membership values $\bar{u}_{f_{1}}=\bar{u}_{f_{2}}=1$, and buses $7,10,17,18$, $19,21,23,24$ and 30 are selected as candidates for VAR extension in the test case. The convergence behaviours of ESSA and SA are shown in Figs. 3 and 4, respectively.

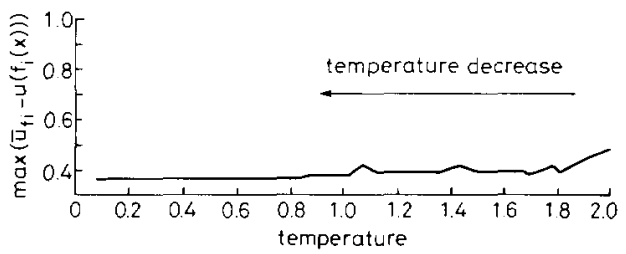

Fig. 3 Converging behaviour of $\min \max \left(\bar{u}_{f_{i}}-u\left(f_{i}(x)\right)\right)$ by ESSA $(30$

The results, before planning and after planning, of ESSA are shown in Table 1, and the performance of ESSA compared with the performance of SA is shown in Table 2. From these results, the algorithm based on the ESSA technique can be faster than the one based on the SA technique and the solution quality is also improved slightly.

384
To evaluate the performance of various distinct membership functions for each objective function, three cases are considered:

Case 1: The membership functions of the cost function $f_{1}$ and the voltage deviation function $f_{2}$ are both linear (see Fig. 1a).

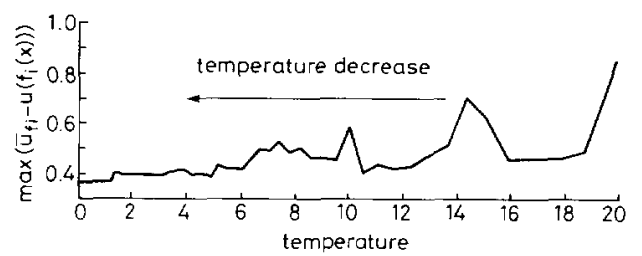

Fig. 4 Converging behaviour of $\min \max \left(\bar{u}_{f_{1}}-u\left(f_{f}(x)\right)\right)$ by $S A$ (30bus)

\begin{tabular}{|c|c|c|c|c|}
\hline & \multirow{2}{*}{$\begin{array}{l}\text { Before } \\
\text { planning }\end{array}$} & \multicolumn{2}{|c|}{ After planning } & \multirow{2}{*}{$\begin{array}{l}\text { Reduction } \\
\text { rates }\end{array}$} \\
\hline & & $\begin{array}{l}\text { Membership } \\
\text { values }\end{array}$ & $\begin{array}{l}\text { Actual } \\
\text { values }\end{array}$ & \\
\hline $\begin{array}{l}\text { Cost* } \\
\text { VD }\end{array}$ & $\begin{array}{l}252.757 \\
-0.114444\end{array}$ & $\begin{array}{l}0.66005 \\
0.63987\end{array}$ & $\begin{array}{l}211.049 \\
0.028007\end{array}$ & $\begin{array}{l}\% \\
16.50 \\
75.53\end{array}$ \\
\hline CPU time & & $45 \mathrm{sec}$ & inds & \\
\hline
\end{tabular}

- million dollars, NTs; VD = voltage deviation (p.u.)

Table 2: Performances of ESSA and SA

\begin{tabular}{lllll}
\hline Cases & Methods & Costs & VD & CPU time \\
\hline & & & & s \\
IEEE & ESSA & 212.04901 & 0.028007 & 45 \\
30-bus & SA & 211.92113 & 0.028306 & 97 \\
\hline
\end{tabular}

ESSA = hybrid expert system-simulated annealing method

$\mathrm{SA}=$ simulated annealing method

Cost $=$ million dollars, $N T S$

$\mathrm{VD}=$ voltage deviation (p.u.)

CPU time on a Twinhead workstation, s

Case 2: The membership functions of $f_{1}$ and $f_{2}$ are linear and exponential (see Fig. 1b), respectively.

Case 3: The membership functions of $f_{1}$ and $f_{2}$ are linear and piecewise-linear (see Fig. 1c), respectively.

In the above cases, the reference membership values are set $\bar{u}_{f_{1}}=\bar{u}_{f_{2}}=1$. The planning results of cases 1,2 and 3 are shown in Tables 3 and 4 . According to these results,

Table 3: Planning results of cases 1, 2 and 3

\begin{tabular}{|c|c|c|c|c|c|}
\hline Case & $\begin{array}{l}\text { Power } \\
\text { loss }\end{array}$ & VAR cost & Total cost $f_{1}$ & VD, $f_{2}$ & $\begin{array}{l}\text { CPU } \\
\text { time }\end{array}$ \\
\hline & MW & NTS, million & NTs, million & p.u. & s \\
\hline Case 1 & 9.4688 & 3.682291 & 211.049011 & 0.028007 & 45 \\
\hline Case 2 & 9.0588 & 2.194265 & 200.581985 & 0.032947 & 42 \\
\hline Case 3 & 9.1453 & 2.682331 & 202.964401 & 0.031296 & 44 \\
\hline
\end{tabular}

Table 4: Results of installing new VAR sources for cases 1 , 2 and 3

\begin{tabular}{lll}
\hline Case & Locations (buses)/size (Mvar) & $\begin{array}{l}\text { Total locations/ } \\
\text { total size (Mvar) }\end{array}$ \\
\hline Case 1 & $7 / 3,10 / 9,18 / 12,19 / 3,21 /-3,23 / 12,30 / 6$ & $7 / 48$ \\
Case 2 & $7 / 21,18 / 12,23 / 12,30 / 6$ & $4 / 51$ \\
Case 3 & $7 / 18,17 / 3,18 / 12,23 / 12,30 / 3$ & $5 / 48$ \\
\hline
\end{tabular}

we have that (i) there is little difference in the cost values between expoential and piecewise-linear memberships, and (ii) the performance of the linear membership function is poor. So, further studies on selecting suitable

IEE Proc.-Gener. Transm. Distrib., Vol. 142, No. 4, July 1995 
membership functions are required to obtain better solutions.

\section{Conclusions}

In this paper a new fomulation for the VAR source planning problem treated as a constrained, multiobjective and nondifferentiable optimisation problem is presented. We have developed a two-stage algorithm, the hybrid expertsystem/simulated-annealing method. Specifically, the proposed hybrid algorithm has the following characteristics:

(i) Theoretically,the global solution can be achieved with probability ' 1 '. Moreover, in practice, the finite interative solution process can yield a near-global solution.

(ii) It is suited to various diffeentiable or nondifferentiable objective functions with equality and inequality constraints. In addition, it can deal with a mixture of continuous and discrete variables.

(iii) It is simple to implement.

(iv) With various distinct membership functions, the characteristics of the multiobjective functions can be properly expressed.

(v) The solution time is much less than that of the conventional simulated annealing method.

(vi) It is insensitive to the initial starting point and so the quality of the solution is good as well as stable.

\section{Appendix}

HRI: The rule used to select all candidate buses, then put them on CANDIDATE

IF bus $i$ is load bus (PQ bus)

AND satisfies all constraints

THEN puts bus $i$ into CANDIDATE.

HR2: The rule used to select a bus, to be placed in a unit reactive power source, or the bus is eliminated from CANDIDATE

Rule 2.I: Find a bus with the biggest $|S I[i]|$ from CANDIDATE.

Rule 2.2: IF the $S I[i]<0$

AND $V_{i}<V_{i r}$

THEN place a unit capacitor on bus $i$

ELSE IF the $S I[i]>0$

AND $V_{i}>V_{i r}$

THEN place a unit reactor on bus $i$

ELSE bus $i$ is eliminated from CANDIDATE.

IF CANDIDATE is empty

THEN run terminating rule

ELSE run $\mathrm{HR} 2$

Rule 2.3: Calculate objective function

$\mathrm{OBJ}=k_{1}{ }^{*} P_{\text {loss }}$ Reduction $-k_{2}{ }^{*}$ Total $-Q_{\text {source }}$ Cost

where:

$k_{1}=$ annual savings due to real power loss reduction (NTS/kW/year)

$k_{2}=$ annual fixed charge rate

$P_{\text {loss }}$ Reduction $=$ annual real power loss reduction

Total $Q_{\text {source }}-$ Cost $=$ total purchase cost of new installment VAR source.

Rule 2.4: IF OBJ $>0$

AND satisfies all constraints

THEN calculate SI and run HR 1

ELSE don't place reactive power source on the bus and eliminate the bus from CANDIDATE.

Terminating rule: Terminate the search procedure and then output the result. 